\title{
Latexin sensitizes leukemogenic cells to gamma- irradiation-induced cell-cycle arrest and cell death through Rps3 pathway
}

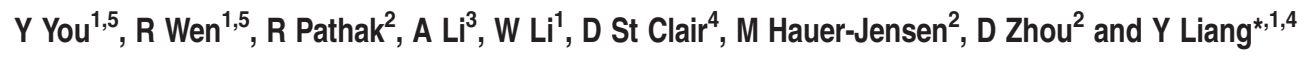

Leukemia is a leading cause of cancer death. Recently, the latexin (Lxn) gene was identified as a potential tumor suppressor in several types of solid tumors and lymphoma, and Lxn expression was found to be absent or downregulated in leukemic cells. Whether Lxn functions as a tumor suppressor in leukemia and what molecular and cellular mechanisms are involved are unknown. In this study, the myeloid leukemogenic FDC-P1 cell line was used as a model system and Lxn was ectopically expressed in these cells. Using the protein pull-down assay and mass spectrometry, ribosomal protein subunit 3 (Rps3) was identified as a novel Lxn binding protein. Ectopic expression of Lxn inhibited FDC-P1 growth in vitro. More surprisingly, Lxn enhanced gamma irradiationinduced DNA damages and induced cell-cycle arrest and massive necrosis, leading to depletion of FDC-P1 cells. Mechanistically, Lxn inhibited the nuclear translocation of Rps3 upon radiation, resulting in abnormal mitotic spindle formation and chromosome instability. Rps3 knockdown increased the radiation sensitivity of FDC-P1, confirming that the mechanism of action of Lxn is mediated by Rps3 pathway. Moreover, Lxn enhanced the cytotoxicity of chemotherapeutic agent, VP-16, on FDC-P1 cells. Our study suggests that Lxn itself not only suppresses leukemic cell growth but also potentiates the cytotoxic effect of radio- and chemotherapy on cancer cells. Lxn could be a novel molecular target that improves the efficacy of anti-cancer therapy.

Cell Death and Disease (2014) 5, e1493; doi:10.1038/cddis.2014.443; published online 23 October 2014

Leukemia is one of the most common cancers and one of the leading causes of cancer death. In the United States, an estimated 52380 new cases of leukemia will be diagnosed in 2014, and approximately 24090 patients will die of this disease ( $\mathrm{NCl}$ Stat Fact Sheets: http://seer.cancer.gov/ statfacts/html/leuks.html). Leukemia can be treated by chemotherapy, radiation therapy and bone marrow transplantation. Radiation and most chemotherapeutic reagents induce apoptosis in tumor cells, resulting in tumor regression. However, some cancer cells are resistant to therapy-induced apoptosis, leading to treatment failure and cancer relapse. ${ }^{1,2}$ There is an urgent need for discovery of novel molecular targets that can abrogate death resistance in leukemic cells and enhance the cytotoxic efficacy of anti-cancer therapies.
Latexin (Lxn) could be an interesting candidate for this purpose. Lxn is a novel regulator of hematopoietic stem cell function and homeostatic hematopoiesis. ${ }^{3}$ It is downregulated in several types of solid and liquid tumors, and overexpression of Lxn inhibits tumor cell growth. ${ }^{4-8}$ In lymphoma, the tumor suppressor function of $\mathrm{Lxn}$ is mediated through $\mathrm{Bcl}-2$-induced apoptosis. ${ }^{5}$ Moreover, Lxn was reported to be a TNFresponsive gene in human papillovirus-infected keratinocytes, suggesting that it may contribute to TNF-induced suppression of cervical cancer. ${ }^{9}$ Lxn is also implicated in inflammation because it is highly enriched in mast cells and can be upregulated by lipopolysaccharide. ${ }^{10,11}$ In addition, Lxn regulates the interaction of hematopoietic stem/progenitor cells to stroma through altering the abundance of cell

\footnotetext{
${ }^{1}$ Department of Internal Medicine, University of Kentucky, Lexington, KY 40536, USA; ${ }^{2}$ Division of Radiation Health, Department of Pharmaceutical Sciences, University of Arkansas for Medical Sciences, Little Rock, AR, USA; ${ }^{3}$ Department of Pathology, Harvard Medical School, Boston, MA 02115, USA and ${ }^{4}$ Gratuate Center for Toxicology, University of Kentucky, Lexington, KY 40536, USA

${ }^{*}$ Corresponding author: Y Liang, Division of Hematology/Bone Marrow Transplantation, Department of Internal Medicine, Markey Cancer Center, University of Kentucky, Room CC408, 800 Rose Street, Lexington, KY 40536-0093, USA. Tel: +1 859323 1729; Fax: +1 859257 7715; E-mail: ying.liang@uky.edu

${ }^{5}$ Co-first authors.

Abbreviations: Lxn, latexin; Rps3, ribosomal protein subunit 3; NCI, National Cancer Institute; TNF, tumor necrosis factor; Bcl-2, B-cell CLL/lymphoma 2; CPA, carboxypeptidase A; DNA, deoxyribonucleic acid; CPA4, carboxypeptidase A4; CPA1, carboxypeptidase A1; TAP, tandem affinity purification; MS, mass spectrometry; GFP, green fluorescent protein; kDa, kilodalton; LC-MS, liquid chromatography-mass spectrometry; p53, tumor protein p53; NF-kB, nuclear factor kappa-light-chainenhancer of activated B cells; Co-IP, complex immunoprecipitation; Gy, gray (unit); VP-16, etoposide phosphate; DSB, DNA double strand break; $\gamma$-H2A.X, phosphorylated H2A histone family, member X; ATM, ataxia telangiectasia mutated; ATR, ataxia telangiectasia and Rad3 related; Chk2, checkpoint kinase 2; Chk1, checkpoint kinase 1; pChk1, phosphorylated checkpoint kinase 1; pChk2, phosphorylated checkpoint kinase 2; BrdU, Bromodeoxyuridine; 7AAD, 7-aminoactinomycin D; G0 phase, gap0/ resting phase; G1 phase, growth 1/gap 1 phase; S phase, synthesis phase; G2 phase, pre-mitotic phase; M phase, mitotic phase; DAPI, 4',6-diamidino-2-phenylindole; RNAi, RNA interference; shRNA, short hairpin RNA; MDM2, mouse double minute 2 homolog; FDC-P1, factor-dependent cell progenitors; DMEM, Dulbecco's Modified Eagle's Medium; FBS, fetal bovine serum; IL-3, interleukin-3; ATCC, American Type Culture Collection; LTR, long terminal repeat; MPSV, myeloproliferative sarcoma virus; SFFV, spleen focus-forming virus; PCR, polymerase chain reaction; Tris-HCl, tris(hydroxymethyl)aminomethane hydrochloride; $\mathrm{NaCl}$, sodium chloride; PMSF, phenylmethylsulfonyl fluoride; SDS-PAGE, sodium dodecyl sulfate polyacrylamide gel electrophoresis; PBS, phosphate-buffered saline; FITC, fluorescein isothiocyanate; $\mathrm{KCl}$, potassium chloride; CTE, chromatid-type exchange; SCU, sister chromatid union; Dimn, double minutes; FACS, fluorescence-activated cell sorting; PAGE, polyacrylamide gel electrophoresis; PVDF, polyvinylidene fluoride; WB, western blot

Received 25.6.14; revised 04.9.14; accepted 08.9.14; Edited by J Chipuk
} 
adhesion molecules. ${ }^{12}$ The only known Lxn binding protein is carboxypeptidase A (CPA), and it inhibits CPA activity, indicating that $L x n$ might be involved in protein degradation and metabolism. ${ }^{10,13-15}$ However, we have already shown that the tumor suppressor function of Lxn is not through the canonical CPA pathway in lymphoma cells. ${ }^{5}$

Currently, the mechanism of action of Lxn in normal and malignant conditions remains unknown, and no reports have been made as to other proteins that could bind to Lxn. In this study, we aimed to discover novel Lxn binding proteins, and evaluate whether Lxn could enhance the cytotoxic effect of radiation and chemotherapeutic agent on leukemic cells. We used myeloid leukemogenic progenitor cell line FDC-P1 as a model system and ectopically expressed Lxn in these cells. ${ }^{16-18}$ Using a protein pull-down assay and mass spectrometry (MS), we identified ribosomal protein subunit 3 (Rps3) as a novel Lxn binding protein. We then examined the response of Lxn-overexpressing FDC-P1 cells to gammairradiation and found that Lxn sensitizes these cells to radiation-induced cell death and inhibits tumor cell growth. FDC-P1 cells with ectopic Lxn expression demonstrate more DNA double-strand breaks (DSBs) upon irradiation, which triggers a dramatic G2/M arrest and blocks G1- and S-phase entry. The abnormal cell-cycle progression results in massive necrosis and depletion of Lxn-overexpressing cells. Mechanistically, the increased level of $L x n$ reduces nuclear translocation of Rps3 upon radiation, which causes abnormal mitotic spindle formation and chromosome instability. Moreover, Rps3 knockdown increases the radiation sensitivity of FDC$\mathrm{P} 1$ cells, confirming that Rps3 is involved in Lxn-mediated radiation response. In addition, Lxn enhances cytotoxicity of chemotherapeutic agent, VP-16, on FDC-P1 cells. This study, for the first time, unravels a mechanistic role of Lxn as a tumor suppressor via a previously unknown Rps3 pathway. Lxn could be a novel molecular target that improves the efficacy of anti-cancer therapy.

\section{Results}

RPS3 is a novel Lxn binding protein. Lxn is the only known CPA inhibitor in mammals; it binds to CPA4 in humans and CPA1 in mouse. ${ }^{10,14}$ We have previously shown that the mechanism of action of $L x n$ is not through inhibition of CPA in lymphoma cells. ${ }^{5}$ Thus, we used the tandem affinity purification (TAP) method in combination with MS to screen novel Lxn binding proteins in FDC-P1 cells, a murine leukemogenic cell line, that can induce myeloid leukemia in vivo (Figure 1a). ${ }^{17,18}$ We first detected the expression of TAPLxn fusion protein with TAP antibody, and found that the fusion protein was expressed only in FDC-P1 cells transduced with TAP-Lxn vector but not with TAP vector (Figure 1b, left panel). By using Lxn antibody, we confirmed overexpression of Lxn protein in TAP-Lxn-transduced cell compared with a very low level of endogenous Lxn in FDC-P1 cells (Figure 1b, right panel). It should be noted that TAP tag itself has $\sim 7.7 \mathrm{kDa}$ molecular weight, thus TAP-Lxn fusion protein is around $35 \mathrm{kDa}$. We next detected proteins differentially present in TAP-Lxn cells and extracted them for LC-MS analysis (Figure 1c). Eleven proteins with significant score were identified, including Lxn itself (Supplementary Table 1). Among these interacting proteins, Rps3 was of particular interest because it has important extra-ribosomal role in DNA damage response and in the regulation of $\mathrm{p} 53$ degradation and NF-KB signaling pathways. ${ }^{19-24}$

We confirmed the expression of Rps3 protein in FDC-P1 cell by western blot (Figure 1d). Protein complex immunoprecipitation (Co-IP) further validates the binding of Lxn and Rps3. The result showed that Lxn was precipitated by Rps3 antibody, and reciprocally, Rps3 was precipitated by Rps3 antibody (Figure 1e, top and bottom panel, respectively). Moreover, immunocytochemical staining result showed that Rps3 and Lxn were colocalized in the cytoplasm (Figure 1f). Therefore, Rps3 is a novel Lxn binding protein and both are localized in the cytoplasm of FDC-P1 cell in non-stressed condition.

Lxn inhibits FDC-P1 cell growth in normal and stress conditions. Rps3 has an important role in DNA damage and repair. ${ }^{21,23,25-29}$ Lxn is a negative regulator of hematopoietic stem and progenitor cell population size.,12,30 We asked whether Lxn overexpression could affect the growth of FDCP1 cells in normal and stress conditions. FDC-P1 cells (TAPLxn or TAP transfected) were irradiated with different doses of gamma-irradiation ( 3 and $6.5 \mathrm{~Gy}$ ), and the cell number was monitored over a period of 9 days of culture after radiation. In the untreated condition, overexpression of Lxn resulted in a 2-fold decrease in the number of FDC-P1 cells (Figure 2; $P=0.007$ at day 5 and $P=0.006$ at day 9 ) although the same number of cells was seeded into the culture at day 0 in both groups. Radiation stress dramatically reduced the cell growth and demonstrated a dose-dependent response. TAP-Lxn cells showed a 2-fold decrease in number at day 5 after 3 Gy irradiation $(P=0.005)$, and it further decreased to 8-fold with the dose of $6.5 \mathrm{~Gy}(P=0.009)$. Lxn-mediated growth suppression was further amplified for the following days of culture and by day 9 nearly 3 -fold fewer cells were present in TAPLxn group than in control group $(P=0.03)$ at 3 Gy. More surprisingly, TAP-Lxn cells were completely depleted by 6.5 Gy irradiation at day 9 , whereas a certain number of control cells were recovered and started to grow.

We next determined cellular response of TAP-Lxn and TAPtransduced FDC-P1 cells to another DNA damage agent, VP-16. VP-16 is an anti-cancer chemotherapy drug and classified as topoisomerase II inhibitor. Figure $2 \mathrm{~b}$ shows that VP-16 dramatically inhibited FDC-P1 growth and had a dosedependent effect in the reduction of cell quantity. At day 9, the number of TAP-Lxn cells was nearly 2 -fold less than control group at the dose of $30 \mu \mathrm{g} / \mathrm{ml}(P=0.005)$. Altogether, $\mathrm{Lxn}$ inhibits myeloid leukemic cell growth and sensitizes them to radiation and chemotherapy-induced cytotoxicity. Since radiation exerts more profound effect on Lxn-overexpressing cells, the following mechanistic study will focus on this condition. It is plausible that the similar mechanism may be applied to Lxnmediated chemo-sensitivity.

Lxn causes chromosomal instability and increases radiation-induced DNA damages. Gamma irradiation causes DNA DSBs. ${ }^{31}$ We asked whether the increased level of DNA damages and/or less efficient repair results in the 


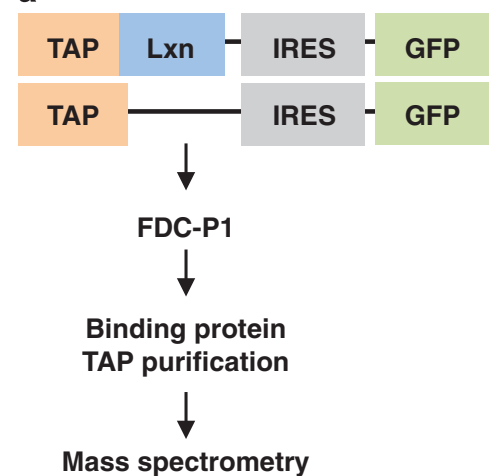

b

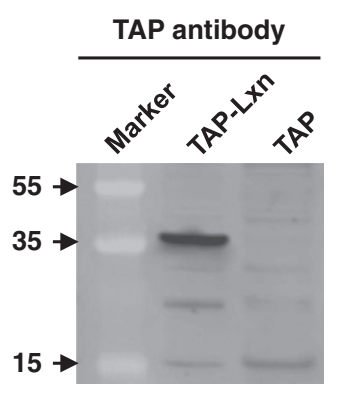

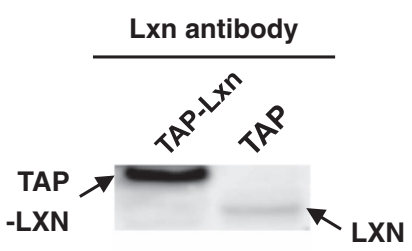

ACT
C

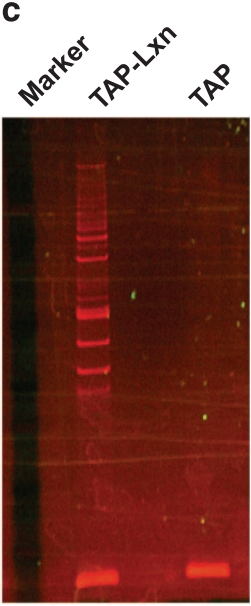

d

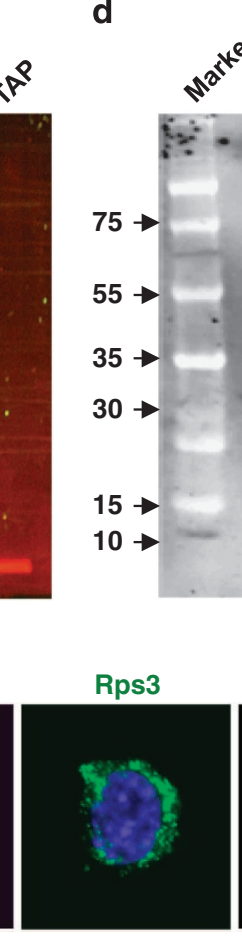

e

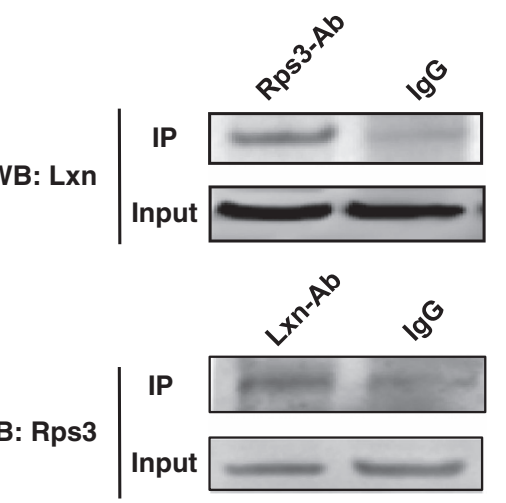

f

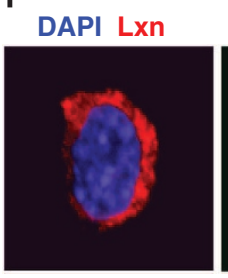

Merge

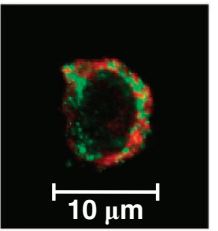

Figure 1 Rps3 is a novel Lxn binding protein. (a) Experimental scheme for isolation of $L x n$ binding protein. Full-length mouse $L x n$ was fused in-frame to an $N$-terminal tandem affinity purification (TAP) tag and sub-cloned into Sf $\beta 9$.1-GFP retroviral vector to create the TAP-Lxn vector. A plasmid carrying the TAP tag was used as a control (TAP). FDC-P1 cells were transfected with the TAP-Lxn or TAP retroviral supernatant, and GFP+ cells were sorted by flow cytometry to establish GFP+ stable cell line. Whole-cell lysates from TAP-Lxn or TAP FDC-P1 cells were purified with the InterPlay Mammalian TAP purification kit and the differentially expressed bands were subjected to mass spectrometry. (b) Detection of TAP-Lxn or TAP protein expression in stable GFP+FDC-P1 by antibodies against TAP tag (left panel) and Lxn (right panel), respectively. (c) Proteins differentially present in FDC-P1 cells transfected with TAP-Lxn or TAP vectors. (d) Rps3 protein is expressed in FDC-P1 cells. Western blot was performed with anti-Rps3 antibody. (e) Co-immunoprecipitation confirms the binding of Lxn and Rps3. Whole-cell lysate from FDC-P1 cells was incubated with Rps3 antibody or lgG control, and the precipitated proteins were probed with anti-Lxn antibody in western blot (WB:Lxn) (top panel). The binding was confirmed by the reciprocal way in which the protein complex was precipitated by Lxn antibody and then western blotted with Rps3 antibody (WB: Rps3) (bottom panel). (f) Lxn and Rps3 are colocalized in the cytoplasm of FDC-P1 cells. FDC-P1 cells were fixed and stained with immunofluorescent probes for Lxn and Rps3 proteins. Lxn was detected by polyclonal antibody and rhodamine-conjugated goat-anti-chicken secondary antibody; Rps3 was detected by polyclonal antibody and FITC-conjugated goat anti-rabbit secondary antibody. Scale bar represents $10 \mu \mathrm{m}$

radio-sensitivity of Lxn-overexpressing FDC-P1 cells. We measured the dynamic formation of phosphorylated H2A.X $\left(\gamma\right.$-H2A.X), a marker for DNA DSB, ${ }^{32,33}$ in FDC-P1 cells after a low dose of $2 \mathrm{~Gy}$ irradiation. Figure $3 \mathrm{a}$ shows that the number of $\gamma$-H2A.X foci at a single cell level was significantly increased at early time point $(1 \mathrm{~h})$ post irradiation in TAP and TAP-Lxn cells but no significant difference was observed between two groups. However, TAP-Lxn cells demonstrated a significantly higher number of $\gamma-\mathrm{H} 2 \mathrm{~A}$.X foci than control cells from $3 \mathrm{~h}$ (Figures $3 \mathrm{a}$ and $\mathrm{b}$ ) to $24 \mathrm{~h}$ post irradiation. These data suggest that Lxn may be involved in DSB repair, and increased level of Lxn attenuates the repair leading to accumulation of DNA damage.

We next examined expression of proteins involved in DNA damage response, including ATM, ATR, phosphorylated Chk2 and Chk1, and p53. Although ATM-Chk2 pathway has a predominant role in cellular response to radiation, we did not detect any significant difference in the expression of ATM and 

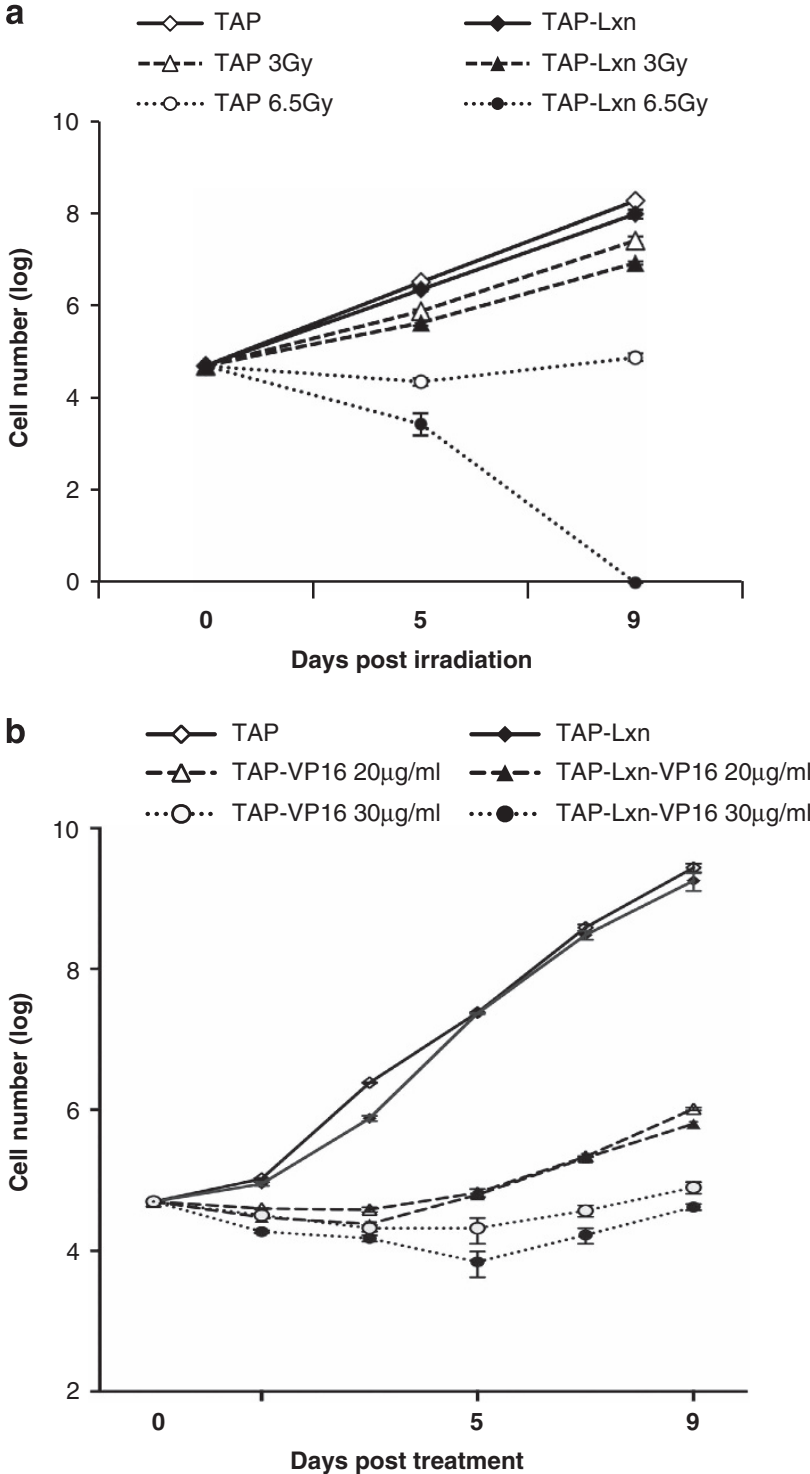

Figure 2 Lxn potentiates cytotoxicity of radiation and chemotherapy drug. (a) Lxn increases radiation sensitivity of FDC-P1 cells and inhibits their growth in a dosedependent manner. FDC-P1 cells transduced with TAP-Lxn or TAP vector were treated with different doses of radiation ( 3 or 6.5 Gy). (b) Lxn enhances cytotoxicity of chemotherapy drug. FDC-P1 cells transduced with TAP-Lxn or TAP vector were treated with different doses of VP-16 (20 or $30 \mu \mathrm{g} / \mathrm{ml})$. Cell growth was measured by trypan blue cell count at indicated time points for 9 days. The cell number was represented by log scale. Three independent experiments were performed and the data were presented by the average \pm standard deviation (S.D.). $P$-values at each time point were indicated in the text

phosphorylated Chk2 between TAP-Lxn and TAP cells at $4 \mathrm{~h}$ post 6.5 Gy irradiation (data not shown). Instead, we found that the protein level of ATR and phosphorylated Chk1 was lower in TAP-Lxn cells than in control cells in non-irradiated condition, and upon radiation, the activation of ATR-Chk1 axis was significantly inhibited in TAP-Lxn cells (Figure 3c). This result suggests that increased level of Lxn could inhibit ATR-Chk1 pathway, resulting in a deficient surveillance mechanism. These changes could cause chromosomal instability in nonstress condition and accumulation of more DNA DSBs upon radiation. To confirm the chromosomal instability in non-stress condition, we performed the cytogenetic study to quantify chromosomal aberrations. Figure $4 \mathrm{~A}$ is the photomicrograph showing different structural aberrations in FDC-P1 cell. Figure 4B summarizes the quantification of each type of aberrations out of total $\sim 300$ cells, and shows that TAP-Lxn cells had a significant higher percentage of aberrant chromosomes than control cells $(13.7 \pm 2.3$ versus $6.4 \pm 1.4$, $P<0.01)$.

In addition, we found that FDC-P1 cells did not express another critical checkpoint protein, p53, which was consistent with the literature (data not shown) ${ }^{34}$ Altogether, Lxn impairs DNA repair capacity and suppresses DNA damage response, leading to accumulation of unrepaired DNA damages in Lxn-overexpressing cells. These cells will probably bypass cell-cycle checkpoint due to lack of p53, accumulate in the later phase of cell cycle, and ultimately undergo massive cell death.

Lxn alters cell-cycle progression and induces cell-cycle arrest. To determine the role of Lxn in DNA damage response, we studied the cell-cycle progression with BrdU and 7AAD using flow cytometry (Figure $5 \mathrm{a}$ ). In the nonirradiated condition $(0 \mathrm{~h})$, Lxn altered the cell-cycle distribution as follows (Figure $5 \mathrm{~b}$ ): the fraction of G0/G1 population in TAP-Lxn cells was half of TAP control cells ( 25 versus $50 \%$ ), and correspondingly nearly 4-fold more TAP-Lxn cells were present in G2/M phase (27 versus 7\%). After irradiation, the fraction of cells in the G2/M phase significantly increased at all time points but the increase was even higher in TAP-Lxn group than in control. At $24 \mathrm{~h}$, nearly $90 \%$ of TAP-Lxn cells were present in the G2/M phase whereas around $50 \%$ of TAP control cells were in this phase. The increased population of G2/M-phase cells corresponded well with the reduced population of G0/G1 cells, suggesting that Lxn prevents cells from returning to the G0/G1 phase, thus blocking normal cellcycle progression. As a result, TAP-Lxn cells demonstrated a dramatic decrease in the fraction of $S$ phase after irradiation. At $24 \mathrm{~h}$, only $6.2 \%$ of cells held at $\mathrm{S}$ phase compared with control $(23.5 \%)$, and a nearly complete depletion of $\mathrm{S}$ population in TAP-Lxn cells at 48 and $72 \mathrm{~h}$. Thus, radiationinduced cell-cycle arrest and depletion of proliferating cells may contribute to the elimination of Lxn-overexpressing cells (see Figure 2a).

Lxn potentiates radiation-induced cell death. To determine whether the abnormal cell-cycle progression and accumulation of DNA damages in Lxn-overexpressing FDCP1 cells could lead to cell death, we used Annexin V and 7$A A D$ staining and flow cytometry to determine apoptosis and necrosis. Upon 6.5 Gy irradiation, TAP-Lxn cells directly underwent necrosis without initiating early apoptotic response, whereas a proportion of control cells was apoptotic (Figure 6a). At all detected time points, nearly 2 -fold more TAP-Lxn cells were necrotic than control cells, which may explain the rapid clearance of these cells during 9 days of cell culture (see Figure 2a). Such different cell death response was also confirmed at the molecular level. Caspase-3, a primary executioner of apoptosis, was not activated in TAPLxn cells but its active form was detected in control cells from $24 \mathrm{~h}$ post irradiation (Figure $6 \mathrm{~b}$ ). These data suggest that $\mathrm{Lxn}$ is involved in the regulation of cell death response. 


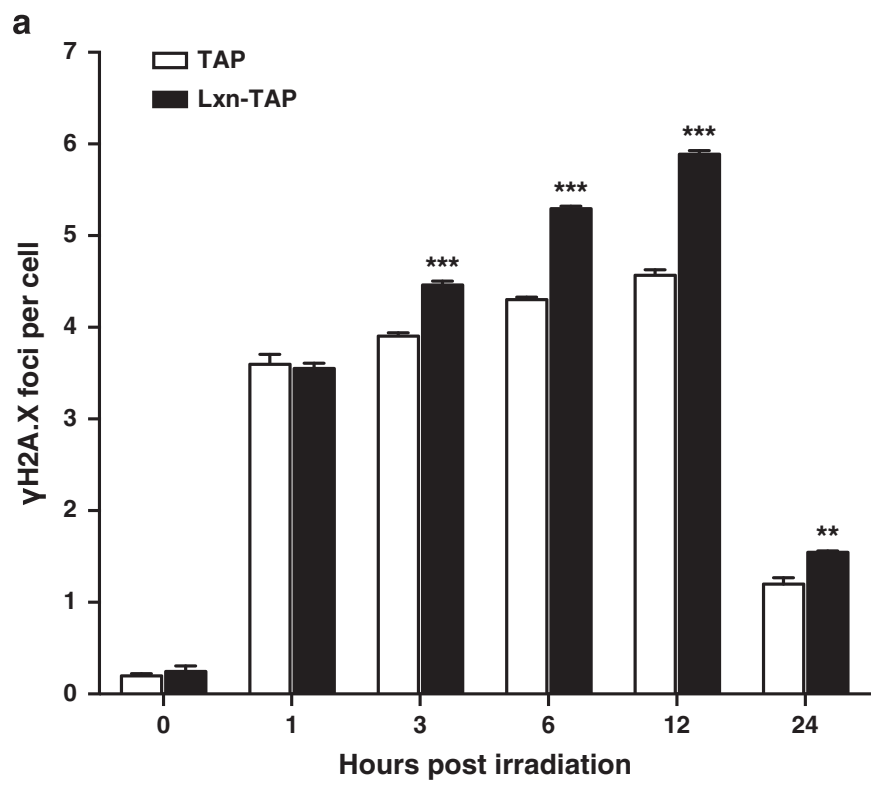

b

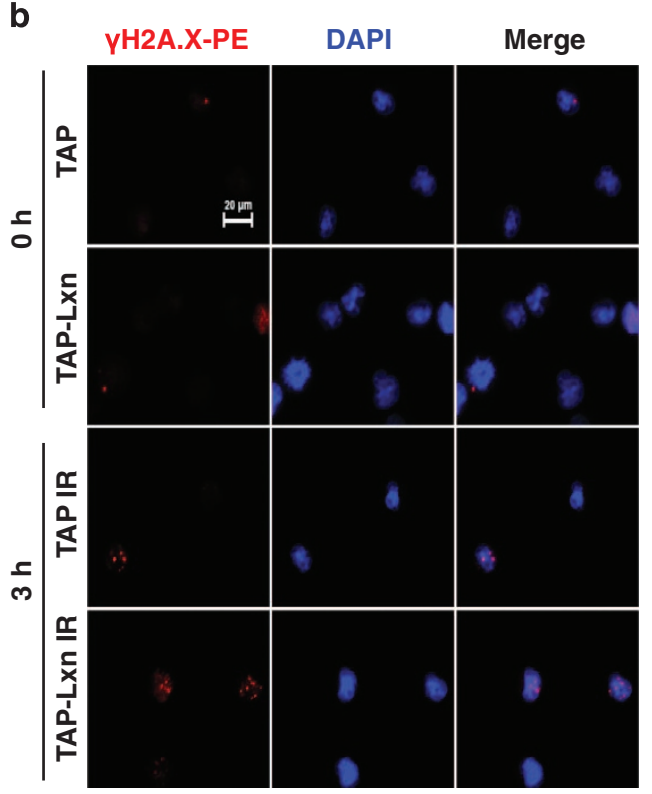

C

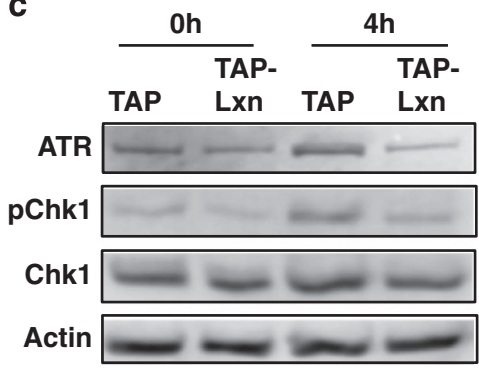

Figure 3 Lxn increases radiation-induced DNA damages and inhibits DNA damage response checkpoints. (a) Lxn-overexpressing FDC-P1 cells had more radiation-induced DNA DSB). FDC-P1 cells transfected with TAP-Lxn or TAP control vectors were irradiated at a dose of 6.5 Gy and DSBs were detected by $\gamma$-H2A.X-PE staining for the formation of $\gamma$-H2A.X foci during $24 \mathrm{~h}$ post irradiation. Y axis represents the average number of $\gamma$-H2A.X foci per cell out of $60-80$ cells. ${ }^{* \star} P<0.01$ and ${ }^{* \star *} P<0.001$. (b) Representative immnunofluorescent imaging of $\gamma$-H2A.X foci in FDC-P1 cells at $3 \mathrm{~h}$ post irradiation. (c) Lxn inhibits the activation of ATR-Chk1 pathway. Expression of ATR and native and phosphorylated Chk1 (pChk1) was detected by western blot in TAP-Lxn and TAP cells at $4 \mathrm{~h}$ post 6.5 Gy irradiation

Lxn inhibits nuclear translocation of Rps3 upon irradiation and induces abnormal mitotic spindle formation. Rps3 is located in the cytoplasm of FDC-P1 cells (see Figure 1f). It was reported that Rps3 translocates into the nucleus upon DNA damage stress; ${ }^{35}$ therefore, we asked whether or not the nuclear translocation of Rps3 happens in Lxn-overexpressing FDC-P1 cells and how the increased level of Lxn affects this process. Immunohistochemical staining of Rps3 at $4 \mathrm{~h}$ post $6.5 \mathrm{~Gy}$ irradiation shows that Rps3 was translocated to the nucleus in both TAP-Lxn and control cells (Figure 7a). This result was further confirmed by western blot of Rps3 in the nuclear and cytoplasm protein lysates of both types of cells (Figure 7b). More interestingly, the amount of translocated Rps3 was significantly less in TAP-Lxn cells than in control (Figure $7 \mathrm{~b}$, top panel with red highlight), suggesting that $L x n$ inhibits nuclear translocation of Rps3 upon irradiation. It was reported that Rps3 facilitates spindle formation and chromosome movement during mitosis. ${ }^{36}$ We thus hypothesized that the reduced amount of nuclear Rps3 might interfere with spindle formation. By using anti-tubulin antibody and DAPI to immunohistochemically stain spindle and chromosome at 12 and $24 \mathrm{~h}$ after 
A
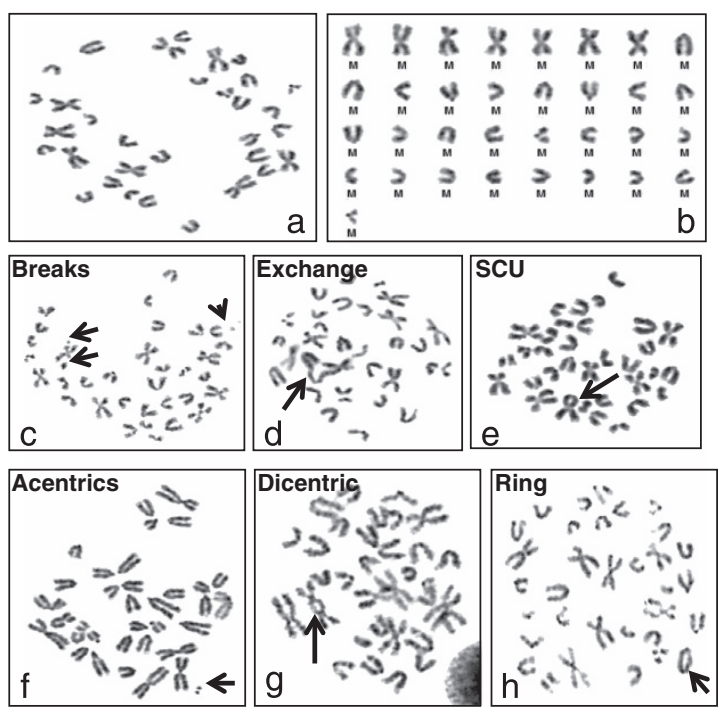

B

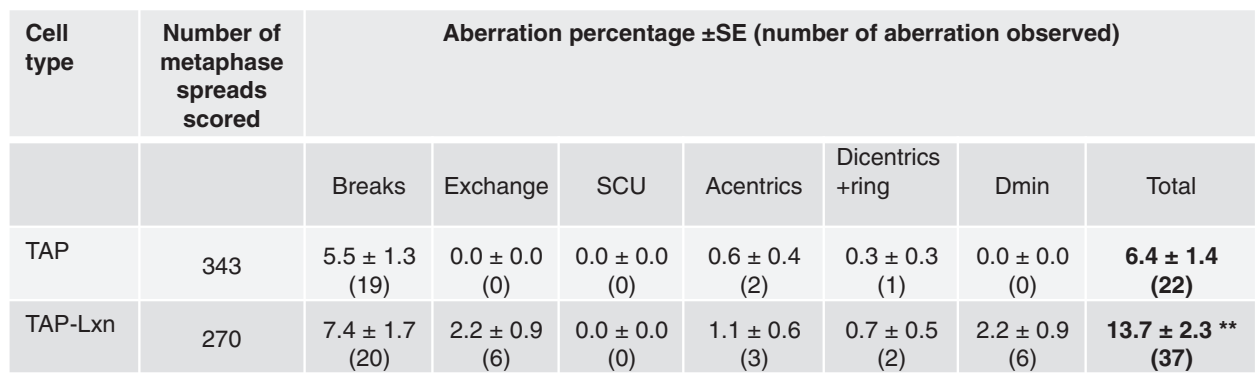

Figure 4 Lxn increases chromosomal instability. (A) Representative photomicrograph showing different structural chromosomal aberrations. Arrows indicate (a) normal metaphase spread with 33 chromosomes, (b) karyotype of the normal metaphase showing seven metacentric chromosomes, (c) chromatid-type breaks, (d) chromatid-type exchange, (e) sister chromatid union, $(\mathrm{f})$ acentric fragment, $(\mathrm{g})$ dicentric chromosome and $(\mathrm{h})$ ring chromosome. (B) Lxn increases chromosomal aberrations. Aberration percentage \pm S.E. (total number of aberration observed) detected in TAP control and TAP-Lxn FDC-P1 cells. Standard errors on the aberration percentage were calculated by $\sqrt{ } a / A$, where $a$ is the number under consideration and $A$ is the total number of cells analyzed. The abbreviations used for chromosomal aberrations are according to international nomenclature. ${ }^{47}$ Z-test was used for group comparison. ${ }^{46 *} P<0.01$

6.5 Gy irradiation, we found that TAP-Lxn cells failed to form the normal mitotic spindle and could not complete the metaphase and the anaphase (Figure 7c). This could explain the cell-cycle arrest in G2/M phases (see Figure 5) as well as the increased chromosomal abnormality (see Figure 4).

Rps3 knockdown increases radiation sensitivity and phenocopies radiation response of Lxn-overexpressing cells. To further confirm that role of $L x n$ in radiation response is mediated by inhibition of Rps3 activity, we knocked down Rps3 in FDC-P1 cells and examined its effect on cell growth and radiation response. It was reported that knocking down Rps 3 protein by $<50 \%$ would not adversely affect ribosomal instability and protein synthesis. The reason is that RNAi knockdown has a specific effect on cytosolic and ribosomefree Rps3, but not the ribosome-bound form. ${ }^{20,22,25}$ Therefore, we chose a shRNA sequence with $\sim 33 \%$ knockdown of Rps3 in FDC-P1 cells (Figure 8a) and established a stable Rps3 knockdown cell line (TAP-Rps3 shRNA). The growth study shows that in comparison with control, the number of TAP-Rps3 shRNA cells was significantly decreased to a level similar to TAP-Lxn cells within 1 day of culture $(P<0.01$, Figure $8 b$ ), suggesting that Rps3 knockdown mimics the effect of Lxn overexpression. With $6.5 \mathrm{~Gy}$ irradiation, the growth of Rps3 knockdown cells was significantly inhibited compared with control although its number was higher than TAP-Lxn cells at day 3 post irradiation. This is probably due to the extent of Rps3 knockdown is not sufficient enough to suppress Rps 3 translocation, thus radiation toxicity is not as significant as Lxn overexpression. These data suggest that RPS3 knockdown increases radiation sensitivity and phenocopies the effect of $L x n$ overexpression.

\section{Discussion}

Lxn is a negative regulator of hematopoietic stem cell numbers. $^{3,30}$ Recent reports have demonstrated that Lxn expression is absent or downregulated in several types of cancers and that Lxn overexpression inhibits tumor growth, suggesting that it may function as a tumor suppressor. ${ }^{4-8,11}$ Caboxypeptidase $\mathrm{A}$ is the only known Lxn binding protein, but we previously showed that the tumor suppressor activity of Lxn 

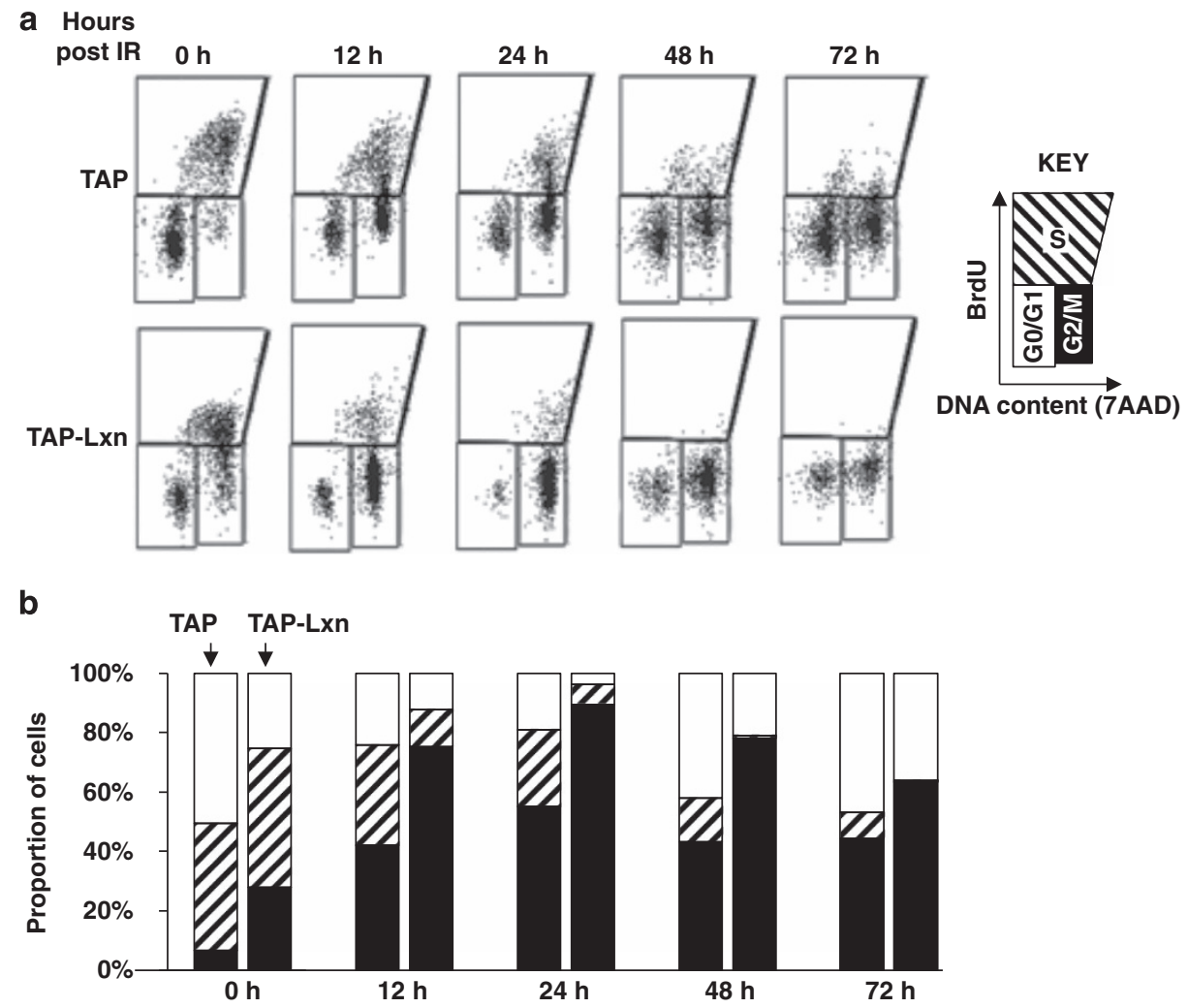

Figure 5 Lxn sensitizes FDC-P1 cells to radiation-induced cell-cycle arrest. Lxn enhances the radiation-induced cell-cycle arrest at G2/M phase and blocks the entry of G1 and S phases. TAP-Lxn or TAP-transfected FDC-P1 cells were treated with 6.5 Gy irradiation and the cell-cycle progression was analyzed by BrdU and 7AAD staining and flow cytometry at indicated time points. The representative flow cytometry profile out of three independent experiments was shown in (a) and quantification of different phases of the cell cycle (G0/G1, S and G2/M phases) was shown in (b)

is not mediated via this canonical pathway. ${ }^{5,10,14}$ In this study, we examined the role of Lxn in myeloid leukemia, elucidated the underlying molecular mechanism, and evaluated its therapeutic potential.

Here, for the first time, we identify Rps3 as a novel Lxn binding protein. Rps3 is a component of the 405 ribosomal subunit and participates in ribosome biogenesis and protein synthesis. Recent reports have revealed its important extraribosomal functions, including DNA damage and repair, ${ }^{29,37}$ induction of apoptosis, ${ }^{23}$ regulation of mitosis, ${ }^{36}$ and involvement in the MDM2-p53 and NF-kB signaling pathways. ${ }^{20,38}$ Some of these biological functions are involved in the nuclear translocation of Rps3. However, the precise role of nuclear Rps3 in DNA damage and response is not yet clear. It can either facility repair due to its endonuclease activity ${ }^{23}$ or block the access of repairing machinery at sites of DNA damage, thus inhibiting repair. ${ }^{21}$ Nuclear Rps3 was also reported to associate with the mitotic spindle and regulate mitosis. ${ }^{36}$ In this study, we found that Lxn and Rps3 are located in the cytoplasm and interact with each other in the unstressed condition. With radiation-induced genotoxic stress, Rsp3 translocates into the nucleus, a result consistent with the previous report. ${ }^{21,35}$ Surprisingly, Lxn attenuates this process, resulting in less Rps3 moving to the nucleus upon irradiation. In addition, Lxn-overexpressing cell demonstrated abnormal mitotic spindle formation. Taken all these evidence together, we propose a model for the mechanism of action of Lxn in radiation response: Lxn sequesters Rps3 in the cytoplasm, resulting in less Rps3 translocation into the nucleus upon irradiation. Reduced level of nuclear Rps3 interferes with formation of the mitotic spindle, induces mitotic abnormalities and blocks cell-cycle progression.

To further address the role of Lxn-Rps3 interaction in cellular response to radiation, it would be necessary to manipulate Rps3 expression and examine its effect on radiation response. Since our data suggest that Lxn inhibits Rps 3 activity (nuclear translocation), we first overexpressed Rps3 in Lxnoverexpressing FDC-P1 cells and found that high level of Rps3 severely suppressed cell growth, which precluded us from further assessing their radiation sensitivity (data not shown). This result is consistent with the previous report in which overexpression of Rps3 can induce apoptosis. ${ }^{21}$ Next, we employed knockdown of Rps3 in FDC-P1 cells to determine whether it can phenocopy Lxn-overexpressing cells. We found that $>60 \%$ knockdown efficiency also produced cytotoxicity, which is consistent with the literature (data not shown). ${ }^{20,22,25}$ These findings are not surprising considering the important function of Rps3 in ribosomal assemble and protein synthesis. Therefore, we finally choose a Rps3 shRNA sequence that can knockdown Rps3 expression by $33 \%$ without direct cytotoxicity. We found that knockdown Rps3 can increase the radiation sensitivity and phenocopy Lxn-overexpressing cells, suggesting that Rps3 might participate in the Lxn-mediated cellular response to 
a

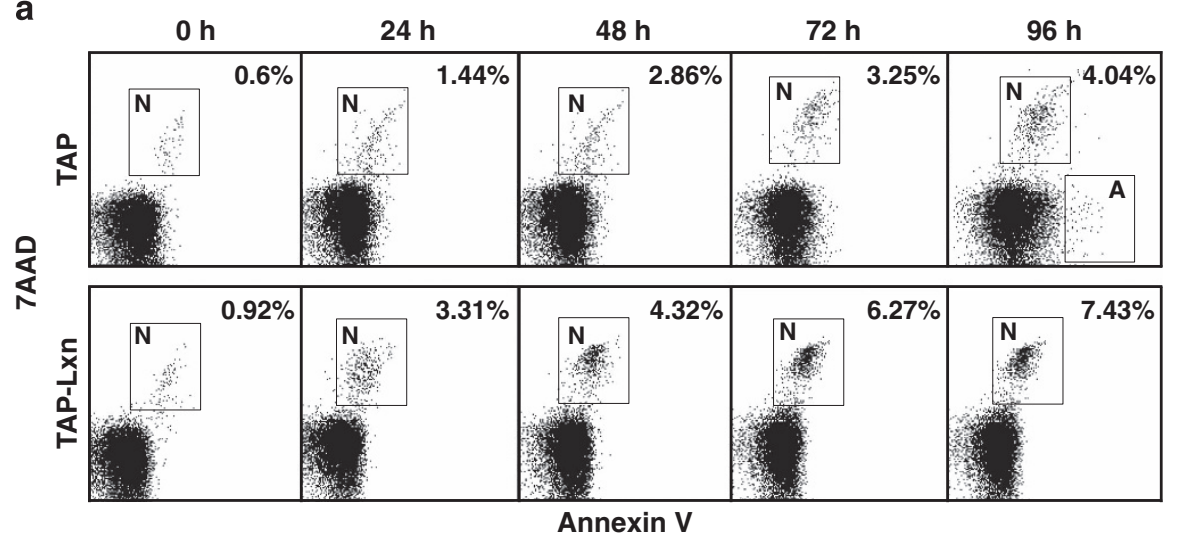

b

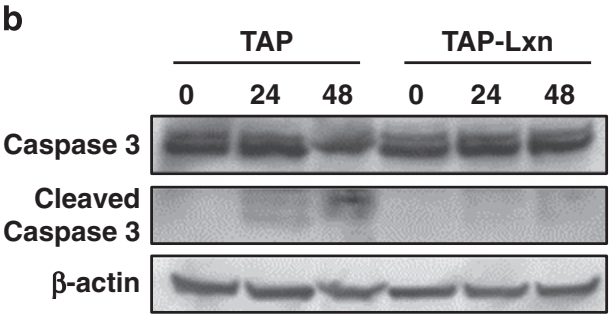

Figure 6 Lxn enhances radiation-induced necrosis. (a) Lxn-overexpressing FDC-P1 cells undergo necrosis in response to radiation. TAP-Lxn or TAP-transfected FDCP1 cells were treated with 6.5 Gy radiation and cell death was determined by Annexin V and 7AAD staining and flow-cytometry analysis. Annexin V-positive and 7AAD-negative cells are apoptotic (labeled as A) and cells positive for both makers are necrotic (labeled as N). Representative flow-cytometry profiles were shown and percentages of apoptotic and necrotic cells at indicated time points post irradiation were labeled. (b) Lxn-overexpressing FDC-P1 cells lack the activation of caspase 3 upon irradiation. Western blot was performed with the antibody against caspase 3 to detect intact and active (cleaved) forms of caspase 3 . $\beta$-Actin was used as the control

radiation. It should be admitted that the ideal strategy to address this question is to block the binding of Lxn and Rps3 and test its functional effect. One of the future studies will be focused on determining their binding domains, designing mutants that interrupt the interaction, and examining their functional effects.

Ectopic expression of Lxn suppresses FDC-P1 growth in vitro, suggesting its potential tumor suppressor function in myeloid leukemia. More importantly, we, for the first time, revealed that $L x n$ sensitizes leukemic cells to radiation and chemo agent-induced cytotoxicity in a dose-dependent manner, and at a certain dose it could completely eliminate cancer cells. Radiation or chemo agents induce DNA damage, and in normal cells, the DNA damage response primarily relies on the ATM-Chk2 signaling, and secondarily on the ATR-Chk1 pathway. ${ }^{39}$ Activation of these pathways will arrest normal cells in G1 via p53-dependent mechanism to allow time for DNA repair or they proceed to cell death if the damage cannot be repaired. ${ }^{40}$ In contrast, many cancers rely heavily on ATRChk1 mediated cell-cycle arrest in the S and G2 phases especially if cancers are deficient in p53. ${ }^{41}$ In consistence with these reports, our result showed that FDC-P1 cell is absent in p53 protein, thus its radiation response is specifically dependent on ATR-Chk1 signaling but not on ATM-Chk2 pathway. Very interestingly, Lxn inhibits the expression of ATR and phosphorylated Chk1 proteins in non-stress condition, resulting in accumulation of aberrant chromosomal structures in Lxn-overexpressing cells. Upon radiation, activation of ATRChk1 was further suppressed in Lxn-overexpressing cells, leading to the abrogation of S/G2 checkpoints and M-phase arrest. Because of the disturbed mitotic spindle, Lxnoverexpressing cells cannot pass through the $M$ phase and ultimately undergo mitotic catastrophe and cell death. p53 mutations occur in approximately $50 \%$ of cancers, which make cancer cells less responsive to the genotoxic agents. ${ }^{42}$ Indeed, leukemia patients with p53 deficiency represent a group with a high resistance to radio- and chemotherapy and demonstrate the most aggressive disease courses. ${ }^{43}$ Therefore, activation of $L x n$ could sensitize p53-deficient tumor cells to radiation and chemotherapeutic agent, and potentiate their cytotoxicity, thus enhancing the efficacy of cancer treatment and reducing the relapse. It was reported that Lxn can be induced by clinically used retinoic acid ${ }^{44}$ and demethylating agent, ${ }^{5}$ suggesting the potential pharmaceutical methods to increase the abundance of Lxn in cancer patients.

Cancer is generally considered to originate from cancer stem cells, which are more resistant to anti-cancer therapies, leading to cancer relapse. ${ }^{45}$ Considering the regulatory role of Lxn in hematopoietic stem cells, we hypothesize that Lxn can be a promising molecular target, in combination with conventional anti-cancer therapy, to improve treatment efficacy and perhaps eradicate cancer stem cells. Our ongoing study focuses on its combinatorial role with chemotherapy in the treatment of leukemia and lymphoma as well as solid tumors.

Materials and Methods

Cell culture. Phoenix GP, 293TN and NIH 3T3 cell lines were cultured in Dulbecco's Modified Eagle's Medium (DMEM, Gibco, Grand Island, NY, USA) supplemented with $10 \%$ fetal bovine serum (FBS), $80 \mathrm{U} / \mathrm{ml}$ penicillin, and $80 \mathrm{mg} / \mathrm{ml}$ streptomycin. FDC-P1 cell line was cultured in DMEM supplemented with $25 \%$ 
a
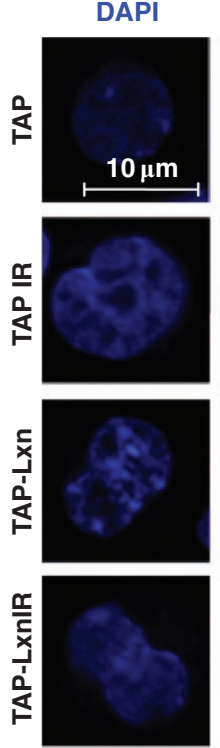

C
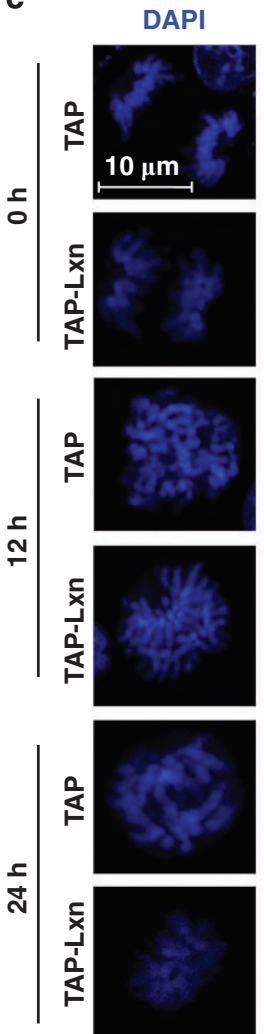

RPS3
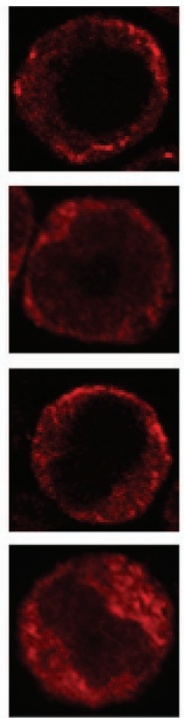

Tublin
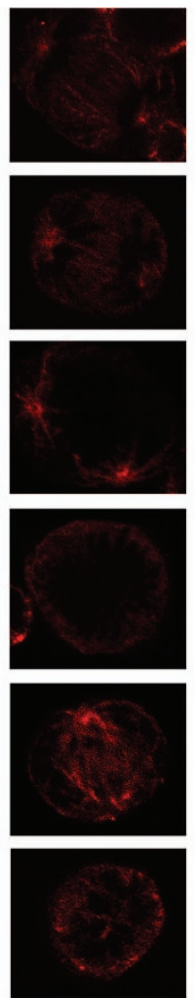

Merge
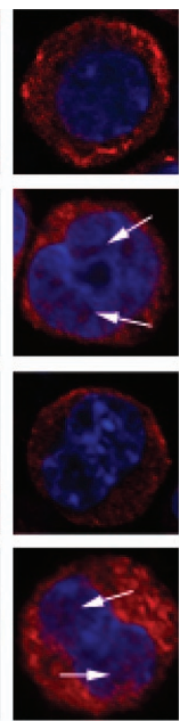

Merge
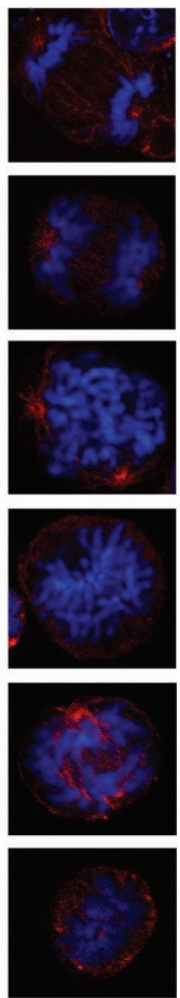

b

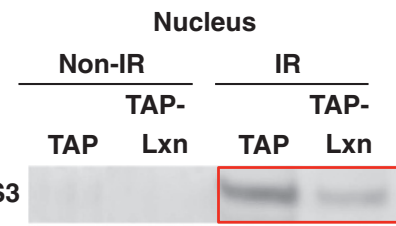

Lamin

$$
\begin{aligned}
& \text { Cytoplasm }
\end{aligned}
$$

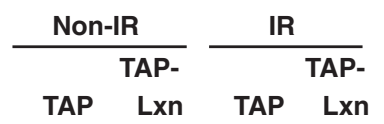

RPS3

Tublin

Figure 7 Lxn attenuates the radiation-induced nuclear translocation of Rps3. (a) Lxn reduces the radiation-induced nuclear translocation of Rps3. TAP-Lxn or TAPtransfected FDC-P1 cells were radiated with a dose of $6.5 \mathrm{~Gy}$ and Rps 3 was immnunohistochemically stained with PE-conjugated Rps 3 antibody before and $4 \mathrm{~h}$ post irradiation. Rps3 was detected in the nucleus only after radiation in both types of cells. (b) Lxn attenuates the nuclear translocation of Rps3. The nuclear and cytoplasm protein of TAP-Lxn or TAP-transfected FDC-P1 cells was extracted. Western blot was performed with Rps3 antibody, and the lamin and actin were used as nuclear and cytoplasm controls, respectively. (c) Lxn interferes mitotic spindle formation. Mitotic spindle and chromosome were immunohistochemically stained with $\alpha$-tublin and DAPI, respectively, at 12 and $24 \mathrm{~h}$ post irradiation, and the representative imaging is shown. Lxn-overexpressing cells could not form the normal spindle and thus the duplicated chromosome failed to segregate and the mitosis was blocked. Scale bar represents $10 \mu \mathrm{m}$

mouse interleukin-3 (IL-3, BD Biosciences, San Jose, CA, USA) and 10\% FBS, $80 \mathrm{U} / \mathrm{ml}$ penicillin and $80 \mathrm{mg} / \mathrm{ml}$ streptomycin. These cell lines were incubated in a humidified atmosphere of $5 \% \mathrm{CO}_{2}$ in air at $37^{\circ} \mathrm{C}$. All cell lines used in this study were purchased from American Type Culture Collection (ATCC).
Construction of overexpression and knockdown vectors and generation of cell lines. The retroviral vector $\mathrm{Sf} \beta 91$ served as a control and the backbone for Lxn overexpression. It contained a 5 ' long terminal repeat (LTR) derived from myeloproliferative sarcoma virus (MPSV) and a 3' LTR derived from 
a
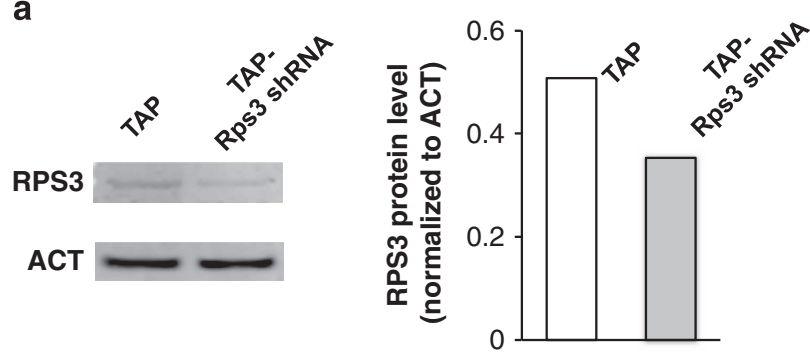

b

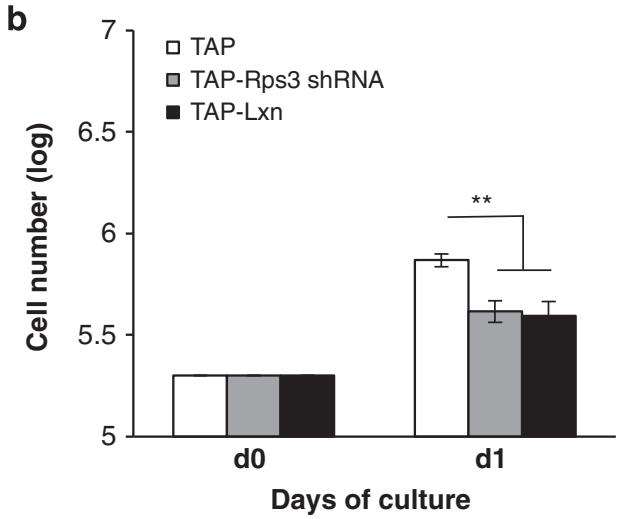

C

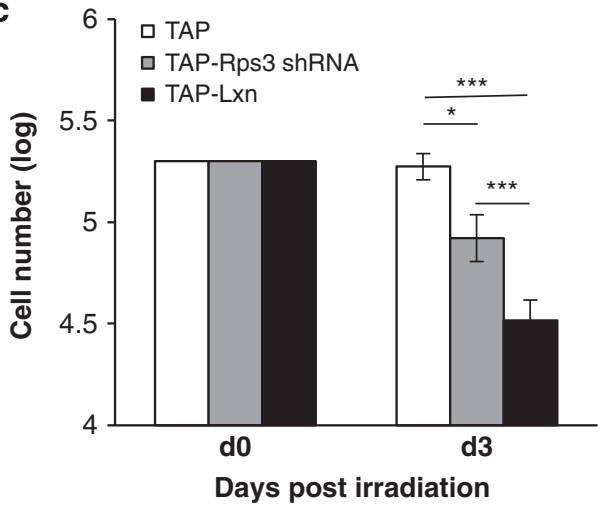

Figure 8 Rps3 knockdown increases radiation sensitivity and phenocopies radiation response of Lxn-overexpressing cells. (a) Knockdown Rps3 in FDC-P1 cells. FDC-P1 cells were transfected with Rps3 shRNA (TAP-Rps3 shRNA) or scramble shRNA (TAP). Rps3 protein level was detected by western blot (left panel) and quantified for knockdown efficiency (right panel). (b) Rps3 knockdown inhibits FDC-P1 growth and phenocopies the effect of Lxn overexpression on FDC-P1 cells (TAP-Lxn) in non-irradiation condition. (c) Rps3 knockdown increases the radiation sensitivity of FDC-P1 cells and produces a similar cytoxicity as Lxn overexpression. FDC-P1 cells with different treatments were subjected to 6.5 Gy irradiation and the cell growth was monitored for 3 days. The absolute numbers of FDC-P1 cells in different treatments were counted as previously described and represented as the average ( \pm S.D.) out of three independent experiments. ${ }^{* \star} P<0.01$. ${ }^{* \star \star} P<0.001$

spleen focus forming virus (SFFV). The internal ribosomal entry site sequence derived from the encephalomyocarditis virus was used for simultaneous translation of the gene insert and enhanced green fluorescent protein (GFP) gene. Full-length mouse Lxn was fused in-frame to a N-terminal TAP tag and subcloned into the Notl site of retroviral vector $\mathrm{s} f \beta$ to create $\mathrm{sf} \beta$-TAP-Lxn. A plasmid comprising only the TAP tag was used as a control. All of the cloned PCR products and constructed plasmids were sequenced. Phoenix GP packaging cells were transfected to produce retrovirus according to the previously described. FDC-P1 cells were then transfected by the retroviral supernatants of TAP control and sf $\beta$-TAP-Lxn vectors in the presence of $8 \mu \mathrm{g} / \mathrm{ml}$ polybrene (Sigma-Aldrich, St. Louis, MO, USA). GFP+ cells were sorted by flow cytometry to create the stable cell line. For Rps3 knockdown, the Rps3 MISSION shRNA plasmid DNA (TRCN0000104309) and the scramble control plasmid DNA (pLKO.1-puro) were purchased from Sigma-Aldrich. The Rps3 shRNA sequence is 5'-CCGGCAACCAGGACAGAAATCATTACTCG AGTAATGATTTCTGTCCTGGTTGTTTTTG- 3 '. In all, $5 \times 10^{5}$ FDC-P1 cells were infected with lentiviral particles carrying Rps3 shRNA or control sequence in the presence of $8 \mu \mathrm{g} / \mathrm{ml}$ polybrene. After $48 \mathrm{~h}$, puromycin $(2 \mu \mathrm{g} / \mathrm{ml})$ was added into the cell culture for the selection of successfully infected cells. After 3 days of selection, survived cells were collected for the measurement of Rps3 protein level and growth and radiation study.

TAP purification and MS. Whole-cell extracts were prepared from TAP-Lxn and TAP expressing FDC-P1 cells and purified using the InterPlay Mammalian TAP purification kit (Stratagene, Agilent Technologies, Santa Clara, CA, USA) following the manufacturer's protocol. Eluted proteins were separated on a $4-12 \%$ SDS-PAGE gel and visualized by SYPRO-RUBY staining. The differentially expressed-bands were cut into slices and processed for mass spectrometric (MC) analysis. The MC analysis was performed at the University of Kentucky, Center for Structural Biology Protein Core Facility and results were submitted to MASCOT for a database sequence similarity search.

Co-immunoprecipitation analysis. FDC-P1 cells expressing Lxn were harvested and lysed on ice using TNTG lysis buffer $(30 \mathrm{mM}$ Tris- $\mathrm{HCl}, 150 \mathrm{mM} \mathrm{NaCl}$, $1 \%$ Triton X-100 and $10 \%$ glycerol) with a protease inhibitor cocktail (Roche, Indianapolis, IN, USA) and $1 \mathrm{mM}$ PMSF (Sigma-Aldrich). After centrifuging, the lysates were saved and used for immunoprecipitation. Briefly, $5 \mu \mathrm{g}$ of anti-Rps3 antibody or control normal rabbit IgG (Santa Cruz, Dallas, TX, USA) was incubated with cell extracts overnight at $4^{\circ} \mathrm{C}$, and subsequently collected by incubating with protein A/G-agarose beads for $1 \mathrm{~h}$ at $4^{\circ} \mathrm{C}$ with rotation. The precipitates were washed five times with lysis buffer and then subjected to SDS-PAGE and detected by western blotting with chicken polyclonal anti-Lxn antibodies (Abcam, Cambridge, MA, USA). The same procedure was performed in a reciprocal manner in which chicken polyclonal anti-Lxn antibody (Abcam) was used to precipitate binding proteins and Rps3 was blotted by anti-Rps3 antibody.

Immunofluorescence. Immunofluorescence was performed for detecting the localization of $L x n$ and Rps3 as well as for staining $\gamma$-H2A.X and spindle. For $L x n$ and Rps3 staining, the adsorbed FDC-P1 cells were incubated at $4{ }^{\circ} \mathrm{C}$ overnight with PBS containing $5 \%(\mathrm{v} / \mathrm{v})$ normal goat serum, $0.1 \%(\mathrm{v} / \mathrm{v})$ Triton $\mathrm{X}-100$ and polyclonal antibodies against Lxn and Rps3 (diluted 1:500). After treatment with FITC and rhodamine-conjugated secondary antibody (diluted $1: 500$ ) at $23^{\circ} \mathrm{C}$ for $2 \mathrm{~h}$, cells were stained with $\gamma$-H2A.X immunofluorescence staining was performed following the similar protocol. Cells were fixed at $0.5 \mathrm{~h}$ post $6.5 \mathrm{~Gy}$ irradiation and then incubated with the PE-conjugated antibody p-histone H2AX (S139) (Cell Signaling, Danvers, MA, USA) at $37^{\circ} \mathrm{C}$ for $1 \mathrm{~h}$. After washing three times, cells were stained with DAPI and the number of $\gamma$-h2A.X foci was counted. In all, 60-80 cells were counted per group for each experimental treatment. Data were expressed as the average number of $\gamma$-H2A.X foci per cells for each time point. The $\alpha$-tublin antibody (Santa Cruz Biotechnology, Dallas, TX, USA) was used for spindle staining following a similar protocol. DAPI fluorescence signals were either taken with a Zeiss Axiovert-200 microscope using a high-resolution Zeiss digital camera (Carl Zeiss Inc., Jena, Germany) or taken with a FV1000 confocal microscope (Olympus, Center Valley, PA, USA).

Measurement of cell growth. FDC-P1 cells transfected with TAP-Lxn and TAP control vectors were treated with (1) 3 Gy or 6.5 Gy $\gamma$-irradiation or (2) VP-16 (Etoposide, Sigma-Aldrich) at the concentration of 20 or $30 \mu \mathrm{g} / \mathrm{ml}$ for $1 \mathrm{~h}$. In each treatment group, $5 \times 10^{4}$ cells were seeded into 24-well tissue culture plates (BD Falcon, Franklin Lakes, NJ, USA) and at day 0 . The cell numbers were subsequently counted on a hemacytometer using trypan blue dye exclusion at different time points for 9 days. Fresh medium was added at each time point. FACS analysis was performed at each time point to measure the percentage of GFP+ cells.

Chromosome stability analysis. Cells in log phase were cultured in fresh media with colcemid $(5 \mu / / \mathrm{ml}$ of media, stock concentration $10 \mu \mathrm{g} / \mathrm{ml}$, Invitrogen, Life Technologies, Grand Island, NY, USA; Cat. No. 15210-040) for $2 \mathrm{~h}$ before harvest. Chromosome preparation was made according to the standard air drying procedure. The cells were harvested by trypsinization, washed with pre-warmed PBS twice, hypotonically treated $\left(0.56 \% \mathrm{KCl}, 20 \mathrm{~min}\right.$ at $\left.37^{\circ} \mathrm{C}\right)$ and subsequently fixed in freshly prepared acetic acid-methanol $(1: 3)$. At least three changes were given in fixative before the cell suspension was dropped on to a pre-cleaned chilled microscopic glass 
slide and dried at room temperature at least for 1 day before staining. Giemsa staining method was applied for scoring metaphase chromosomes. Structural chromosome aberrations such as breaks, chromatid-type exchange (CTE), sister chromatid union (SCU), acentric fragments (acentrics), double minutes (Dimn), dicentric and ring chromosomes were scored under $\times 63$ magnification. In each datum point, $>150-340$ metaphase spreads were scored. The modal chromosome number of FDC-P1 cell was 33 with 7 metacentric chromosomes. Considering the modal chromosome number 33 , total number of chromosome investigated was calculated by multiplying modal chromosome number with number of metaphase spread scored for each datum point. Induction of total structural aberration between two groups (assuming $33 \times$ number of metaphase spreads scored for the purpose of determining proportion values) was compared using Z-test to find the statistical significance between TAP control and TAPLxn cells. Considering one-sided test, if the Z-value is $>1.64$ or 2.33 , then the difference in induction of structural translocations was considered as statistically significant at $5 \%$ level $(P<0.05)$ or $1 \%$ level $(P<0.01)$, respectively. ${ }^{46}$

Flow-cytometry analysis. Cell-cycle analysis: BrdU staining was conducted using the BrdU Flow Kit (BD Bioscience, San Jose, CA, USA) following the manufacturer's instruction. In brief, $10^{6}$ cells were incubated with BrdU in the culture medium at the final concentration of $10 \mu \mathrm{M}$ for $1 \mathrm{~h}$ and cells were then fixed in $100 \mu$ lBD Cytofix/Cytoperm buffer (BD Bioscience) at RT for 20 min. After washing three times with BD Perm/Wash buffer (BD Bioscience), DNase was added into the resuspended cells at the final concentration of $300 \mu \mathrm{g} / \mathrm{ml}$ at $37^{\circ} \mathrm{C}$ for $1 \mathrm{~h}$. After washing, cells were incubated with PE conjugated anti-BrdU antibody (eBioscience, San Diego, CA, USA) at RT for 20 min. 7AAD (BD Bioscience) was added before flow-cytometry analysis. Apoptosis and necrosis analysis: To determine the profile of cell death after $\gamma$-irradiation, Annexin V staining was conducted at $0,24,48,72$ and $96 \mathrm{~h}$ after $6.5 \mathrm{~Gy} \gamma$-irradiation. Annexin V-PE antibody (BD Bioscience) was used to stain the fixed cells following the manufacturer's protocol. 7AAD was added before flow-cytometry analysis. All flow-cytometry analyses were performed on a FACSAria II (Becton Dickinson Immunocytometry Systems, San Jose, CA, USA).

Western blot. Total protein was extracted following the method of previous study. Cytoplasma and nucleus proteins were extracted separately by the Minute Cytoplasmic and Nuclear Extraction Kit (Invent Biotechnologies, Eden Prairie, MN, USA) following the manufacturer's protocol. For western blot, protein lysates were thawed and mixed with running buffer and a reducing agent (Novex, Life Technologies) according to the manufacturer's instructions and heated at $95^{\circ} \mathrm{C}$ for $5 \mathrm{~min}$. Samples were then separated in denaturing PAGE (Novex, $10 \%$ bis-Tris gel) using the equivalent of $4 \times 10^{5}$ cells per lane at $185 \mathrm{~V}$ for $1 \mathrm{~h}$. Following electrophoresis, samples were then transferred onto a PVDF membrane (Millipore, Billerica, MA, USA) by electro-blotting at $30 \mathrm{~V}$ for $1 \mathrm{~h}$, which were subsequently blocked in 5\% skimmed milk and probed with anti-p-Chk1 (S317) rabbit antibody (Cell Signaling), anti-Chk1 mouse antibody (Santa Cruz), anti-p-Chk2 (Thr68) rabbit antibody (Santa Cruz), anti-Chk2 rabbit antibody (Santa Cruz), anti-ATM rabbit antibody (Santa Cruz), anti-ATR rabbit antibody (Cell Signaling), anti- $\beta$-actin mouse antibody (Sigma-Aldrich), chicken polyclonal anti-Lxn antibodies (Abcam), anti-P53 mouse antibody (Santa Cruz), anti-laminB goat antibody (Santa Cruz), anticaspase-3 rabbit antibody (Cell Signaling) and anti-ribosome protein S3 (Rps3) antibody (Santa Cruz), respectively. Primary antibodies were detected using alkalinephosphatase-conjugated secondary antibodies (Santa Cruz Biotechnology) and electro-chemi-fluorescent (ECF) reagent (Amersham Pharmacia Biotech, Piscataway, NJ, USA) according to the manufacturer's instructions. Blots were then visualized using a Molecular Dynamics STORM 860 system (GE Healthcare Life Sciences, Pittsburgh, PA, USA).

Statistical analysis. Data were examined for homogeneity of variances (F-test), then analyzed by Student's t-test or one-way ANOVA using Tukey's test. Differences were considered as significant at $P<0.05$. All statistical analyses were conducted with a statistical package (SPSS 16.0 for Windows, IBM SPSS Statistics, IBM, Armonk, NY, USA)

\section{Conflict of Interest}

The authors declare no conflict of interest.

Acknowledgements. We thank Dr Sabire Ozcan and Megan Sampley for TAP vectors, Carol Beach for mass spectrometry analysis, B Grimes and C Swiderski for technical assistance, and the Markey Cancer Center's Research Communications
Office for manuscript editing. This study was supported by the National Center for Advancing Translational Sciences, National Institutes of Health, grant number KL2TR000116 (YL), start-up funding from the Markey Cancer Center at the University of Kentucky (YL), National Cancer Institute, grant number 5P30CA177558-02, National Institutes of Health, and the Edward P Evans Foundation.

\section{Author contributions}

YY and RW contribute equally to this manuscript. YY performed western blot and Rps3 immunostaining. RW performed VP-16 treatment, H2A.X immnunostaining and RPs3 knockdown experiments. WL performed radiation response, cell cycle and apoptosis analysis. AL identified Rps3 as the binding protein. RP performed chromosomal instability assay. MH-J, DC and DZ provided advice for the project and reviewed the manuscript. $\mathrm{YL}$ is responsible for the design of the study and writing of this paper.

1. Hosoya N, Miyagawa K. Targeting DNA damage response in cancer therapy. Cancer Sci 2014: 105: 370-388.

2. Lord CJ, Ashworth A. The DNA damage response and cancer therapy. Nature 2012; 481: 287-294.

3. Liang $\mathrm{Y}$, Jansen M, Aronow B, Geiger H, Van Zant G. The quantitative trait gene latexin influences the size of the hematopoietic stem cell population in mice. Nat Genet 2007; 39: 178-188.

4. Li Y, Basang Z, Ding H, Lu Z, Ning T, Wei H et al. Latexin expression is downregulated in human gastric carcinomas and exhibits tumor suppressor potential. BMC Cancer 2011; 11: 121

5. Liu Y, Howard D, Rector K, Swiderski C, Brandon J, Schook L et al. Latexin is down-regulated in hematopoietic malignancies and restoration of expression inhibits lymphoma growth. PLoS One 2012; 7: e44979.

6. Abd Elmageed ZY, Moroz K, Kandil E. Clinical significance of CD146 and latexin during different stages of thyroid cancer. Mol Cell Biochem 2013; 381: 95-103.

7. Muthusamy V, Premi S, Soper C, Platt J, Bosenberg M. The hematopoietic stem cell regulatory gene latexin has tumor-suppressive properties in malignant melanoma. $\mathrm{J}$ Invest Dermatol 2013; 133: 1827-1833.

8. Ni QF, Tian Y, Kong LL, Lu YT, Ding WZ, Kong LB. Latexin exhibits tumor suppressor potential in hepatocellular carcinoma. Oncol Rep 2014; 31: 1364-1372.

9. Termini L, Boccardo E, Esteves GH, Hirata Jr R, Martins WK, Colo AE et al. Characterization of global transcription profile of normal and HPV-immortalized keratinocytes and their response to TNF treatment. BMC Med Genomics 2008; 1: 29.

10. Uratani Y, Takiguchi-Hayashi K, Miyasaka N, Sato M, Jin M, Arimatsu Y. Latexin, a carboxypeptidase $A$ inhibitor, is expressed in rat peritoneal mast cells and is associated with granular structures distinct from secretory granules and lysosomes. Biochem J 2000; 346(Pt 3): 817-826.

11. Aagaard A, Listwan $P$, Cowieson $N$, Huber T, Ravasi T, Wells CA et al. An inflammatory role for the mammalian carboxypeptidase inhibitor latexin: relationship to cystatins and the tumor suppressor TIG1. Structure 2005; 13: 309-317.

12. Mitsunaga K, Kikuchi J, Wada T, Furukawa $\mathrm{Y}$. Latexin regulates the abundance of multiple cellular proteins in hematopoietic stem cells. J Cell Physiol 2012; 227: 1138-1147.

13. Liu Q, Yu L, Gao J, Fu Q, Zhang J, Zhang $P$ et al. Cloning, tissue expression pattern and genomic organization of latexin, a human homologue of rat carboxypeptidase $A$ inhibitor. Mol Biol Rep 2000; 27: 241-246.

14. Pallares I, Bonet R, García-Castellanos R, Ventura S, Avilés FX, Vendrell J et al. Structure of human carboxypeptidase A4 with its endogenous protein inhibitor, latexin. Proc Natl Acad Sci USA 2005; 102: 3978-3983.

15. Mouradov D, Craven A, Forwood JK, Flanagan JU, García-Castellanos R, Gomis-Rüth FX et al. Modelling the structure of latexin-carboxypeptidase $A$ complex based on chemical crosslinking and molecular docking. Protein Eng Des Sel 2006; 19: 9-16.

16. Duhrsen U. In vitro growth patterns and autocrine production of hemopoietic colony stimulating factors: analysis of leukemic populations arising in irradiated mice from cells of an injected factor-dependent continuous cell line. Leukemia 1988; 2: 334-342.

17. Duhrsen U, Metcalf D. A model system for leukemic transformation of immortalized hemopoietic cells in irradiated recipient mice. Leukemia 1988; 2: 329-333.

18. Duhrsen U, Metcalf D. Effects of irradiation of recipient mice on the behavior and leukemogenic potential of factor-dependent hematopoietic cell lines. Blood 1990; 75: 190-197.

19. Stanborough T, Niederhauser J, Koch B, Bergler H, Pertschy B. Ribosomal protein S3 interacts with the NF-kappaB inhibitor IkappaBalpha. FEBS Lett 2014; 588: 659-664.

20. Yadavilli S, Mayo LD, Higgins M, Lain S, Hegde V, Deutsch WA. Ribosomal protein S3: A multi-functional protein that interacts with both $\mathrm{p} 53$ and MDM2 through its $\mathrm{KH}$ domain. DNA Repair 2009; 8: 1215-1224.

21. Hegde V, Yadavilli S, McLaughlin LD, Deutsch WA. DNA repair efficiency in transgenic mice over expressing ribosomal protein S3. Mutat Res 2009; 666: 16-22.

22. Wan F, Anderson DE, Barnitz RA, Snow A, Bidere N, Zheng $L$ et al. Ribosomal protein S3: a $\mathrm{KH}$ domain subunit in NF-kappaB complexes that mediates selective gene regulation. Cell 2007; 131: 927-939. 
23. Jang CY, Lee JY, Kim J. RpS3, a DNA repair endonuclease and ribosomal protein, is involved in apoptosis. FEBS Lett 2004; 560: 81-85.

24. Zhang Y, Lu H. Signaling to p53: ribosomal proteins find their way. Cancer Cell 2009; 16: 369-377.

25. Kim Y, Kim HD, Kim J. Cytoplasmic ribosomal protein S3 (rpS3) plays a pivotal role in mitochondrial DNA damage surveillance. Biochim Biophys Acta 2013; 1833 : 2943-2952.

26. Seong KM, Jung SO, Kim HD, Kim HJ, Jung YJ, Choi SY et al. Yeast ribosomal protein S3 possesses a beta-lyase activity on damaged DNA. FEBS Lett 2012; 586: 356-361.

27. Jang CY, Kim HD, Kim J. Ribosomal protein S3 interacts with TRADD to induce apoptosis through caspase dependent JNK activation. Biochem Biophys Res Commun 2012; 421: 474-478.

28. Lee SB, Kwon IS, Park J, Lee KH, Ahn Y, Lee C et al. Ribosomal protein S3, a new substrate of Akt, serves as a signal mediator between neuronal apoptosis and DNA repair. J Biol Chem 2010; 285: 29457-29468.

29. Kim TS, Kim HD, Kim J. PKCdelta-dependent functional switch of $r p S 3$ between translation and DNA repair. Biochim Biophys Acta 2009; 1793: 395-405.

30. de Haan $\mathrm{G}$. Latexin is a newly discovered regulator of hematopoietic stem cells. Nat Genet 2007; 39: 141-142.

31. Matsudaira H, Furuno I, Ueno AM, Shinohara K, Yoshizawa K. Induction and repair of strand breaks and 3'-hydroxy terminals in the DNA of mammalian cells in culture following gammaray irradiation. Biochim Biophys Acta 1977; 476: 97-107.

32. Paull TT, Rogakou EP, Yamazaki V, Kirchgessner CU, Gellert M, Bonner WM. A critical role for histone H2AX in recruitment of repair factors to nuclear foci after DNA damage. Curr Biol 2000; 10: 886-895.

33. Podhorecka M, Skladanowski A, Bozko P. H2AX phosphorylation: its role in DNA damage response and cancer therapy. J Nucleic Acids 2010; 2010.

34. Chang F, McCubrey JA. P21(Cip1) induced by Raf is associated with increased Cdk4 activity in hematopoietic cells. Oncogene 2001; 20: 4354-4364.

35. Wan F, Weaver A, Gao X, Bern M, Hardwidge PR, Lenardo MJ. IKKbeta phosphorylation regulates RPS3 nuclear translocation and NF-kappaB function during infection with Escherichia coli strain 0157:H7. Nat Immunol 2011; 12: 335-343.

36. Jang CY, Kim HD, Zhang X, Chang JS, Kim J. Ribosomal protein S3 localizes on the mitotic spindle and functions as a microtubule associated protein in mitosis. Biochem Biophys Res Commun 2012; 429: 57-62.

37. Kim J, Chubatsu LS, Admon A, Stahl J, Fellous R, Linn S. Implication of mammalian ribosomal protein S3 in the processing of DNA damage. J Biol Chem 1995; 270: 13620-13629.
38. Wan F, Lenardo MJ. The nuclear signaling of NF-kappaB: current knowledge, new insights, and future perspectives. Cell Res 2010; 20: 24-33.

39. Marechal A, Zou L. DNA damage sensing by the ATM and ATR kinases. Cold Spring Harb Perspect Biol 2013; 5: a012716.

40. Zeman MK, Cimprich KA. Causes and consequences of replication stress. Nat Cell Biol 2014; 16: 2-9.

41. Sperka T, Wang J, Rudolph KL. DNA damage checkpoints in stem cells, ageing and cancer. Nature reviews. Mol Cell Biol 2012; 13: 579-590.

42. Muller PA, Vousden KH. Mutant p53 in cancer: new functions and therapeutic opportunities. Cancer Cell 2014; 25: 304-317.

43. Zenz T, Häbe S, Denzel T, Winkler D, Döhner H, Stilgenbauer S. How little is too much? p53 inactivation: from laboratory cutoff to biological basis of chemotherapy resistance. Leukemia 2008; 22: 2257-2258.

44. Oldridge EE, Walker HF, Stower MJ, Simms MS, Mann VM, Collins AT et al. Retinoic acid represses invasion and stem cell phenotype by induction of the metastasis suppressors RARRES1 and LXN. Oncogenesis 2013; 2: e45.

45. Blanpain C, Mohrin M, Sotiropoulou PA, Passegue E. DNA-damage response in tissuespecific and cancer stem cells. Cell Stem Cell 2011; 8: 16-29.

46. Pathak R, Sarma A, Sengupta B, Dey SK, Khuda-Bukhsh AR. Response to high LET radiation $12 \mathrm{C}$ (LET, $295 \mathrm{keV} /$ microm) in M5 cells, a radio resistant cell strain derived from Chinese hamster V79 cells. Int J Radiat Biol 2007; 83: 53-63.

47. Ritter S, Nasonova E, Scholz M, Kraft-Weyrather W, Kraft G. Comparison of chromosomal damage induced by X-rays and Ar ions with an LET of $1840 \mathrm{keV} /$ micrometer in G1 V79 cells. Int J Radiat Biol 1996; 69: 155-166.

(c) (i) Cell Death and Disease is an open-access journal published by Nature Publishing Group. This work is licensed under a Creative Commons Attribution 4.0 International Licence. The images or other third party material in this article are included in the article's Creative Commons licence, unless indicated otherwise in the credit line; if the material is not included under the Creative Commons licence, users will need to obtain permission from the licence holder to reproduce the material. To view a copy of this licence, visit http://creativecommons.org/licenses/by/4.0 